\title{
Adaptive Sliding Mode Variable Structure Control of Chaos in Permanent Magnet Synchronous Motor
}

\author{
Yue Liu $^{1} *$,Shuo $\mathrm{Zhou}^{2}$ \\ *1. School of Science, Shenyang Jianzhu University, Shenyang 110168, China \\ E-mail:888zhoushuo@163.com \\ 2. Department of Mechanical and Electronic Engineering, Guidaojiaotong Polytechnic Institute,Shenyang 110023, China \\ E-mail:8677730@qq.com
}

Keywords: Permanent magnet synchronous motor(PMSM), Chaotic system, Adaptive sliding mode control

\begin{abstract}
This paper proposes a robust adaptive sliding mode control strategy aiming at the complex chaotic behavior of permanent magnet synchronous motor (PMSM). Using the sliding mode control technique, a time varying surface is constructed. Then an adaptive gain of the robust control law is established considering uncertainties and external interference in PMSM. So the phenomenon of chaos in PMSM system will be eliminated using proposed controller. Finally, stability analysis will be given based on Lyapunov stability theory. Simulation results demonstrate the effectiveness of the proposed adaptive sliding mode control scheme.
\end{abstract}

\section{Introduction}

Chaos phenomenon exists widely in many servo systems, for example induction motor, permanent magnet synchronous motor(PMSM), DC motor and switched reluctance motor. During the past two decades, much research has been focused on analysis and control of chaotic phenomenon in motor drive[1-3]. The chaos phenomena in PMSM system was first discovered by Hemati. Then intensive study of theoretical analysis was introduced in. The chaotic system of PMSM is multivariable, strong coupling and nonlinear. The chaotic behavior in PMSM can destroy the stabilization of the motor even inducing the drive system to collapse. So the control of chaos in the PMSM have been an active research area in the field of nonlinear control of electric motors.

The conventional linear control theory have been unable to meet the requirements of PMSM chaotic system. With the development of chaos control theory, many different control methods of PMSM chaotic systems have been proposed such as decoupling control [4], feedback control[5] ,dynamic surface control [6], sliding mode control [7-9] and adaptive control [10] etc.The first three methods are dependent on the mathematical model of the system. The dynamic performance of system can not be guaranteed when the system parameters change. The changing of parameters even induces the drive system to collapse. Sliding mode controller exists serious chattering phenomenon. The adaptive controller need introduce mechanism of parameter adaptive, which increases the complexity of control. Moreover, the above control strategy can guarantee the stability of the system, but can not guarantee convergence in finite-time. Finite-time stability control contains fractional exponential which has stronger robustness and disturbance resistance compared with non finite-time stability control[11]. In view of the above advantages, the method has been widely applied in the network system, chaotic systems[12] , aviation aircraft, etc. In [13] , a finite time stability control of the unified chaotic systems is proposed, but its stability time is long. In [14], finite time stability control was used to PMSM chaotic systems for the first time. However, the influence of system parameter uncertainties was not taken into account.

In this paper, a robust adaptive sliding mode control design strategy is used to control chaos in permanent magnet synchronous motor drive systems. The proposed scheme will give sufficient consideration to uncertainties and external interference in PMSM. Furthermore, the stability of the proposed method is guaranteed using Lyapunov stability theory. Finally, simulation results demonstrate the effectiveness and robustness against the chaotic in PMSM drive system.

\section{Mathematical Model of Chaotic PMSM Drive System}

The mathematical model of the permanent magnet synchronous motor with smooth air gap is described as follows[15]:

$$
\left\{\begin{array}{l}
\frac{d \omega}{d t}=\sigma\left(i_{q}-\omega\right)-\tilde{T}_{L} \\
\frac{d i_{q}}{d t}=-i_{q}-i_{d} \omega+\gamma \omega+\tilde{u}_{q} \\
\frac{d i_{d}}{d t}=-i_{d}+i_{q} \omega+\tilde{u}_{d}
\end{array}\right.
$$


where $i_{d}, i_{q}$ and $\omega$ are the state variables, which represent $\mathrm{d}$ $\mathrm{q}$ axis currents and motor angular frequency. $\tilde{u}_{d}$ and $\tilde{u}_{q}$ are the $\mathrm{d}-\mathrm{q}$ axis stator voltage. $\tilde{T}_{L}$ denotes the load torque. $\sigma$ and $\gamma$ are non-negative system parameters.

In system (1), the external inputs are set to zero, i.e. $\tilde{T}_{L}=\tilde{u}_{q}=\tilde{u}_{d}=0$, and the system dynamic becomes an unforced system as follows:

$$
\left\{\begin{array}{l}
\frac{d \omega}{d t}=\sigma\left(i_{q}-\omega\right) \\
\frac{d i_{q}}{d t}=-i_{q}-i_{d} \omega+\gamma \omega \\
\frac{d i_{d}}{d t}=-i_{d}+i_{q} \omega
\end{array}\right.
$$

The study found that the PMSM experiences chaotic behavior when the system parameters $\sigma$ and $\gamma$ fall in a certain range of values. For example, the PMSM displays chaos with $\sigma=5.45$ and $\gamma=20$.A typical chaotic attractor is shown in Fig.1. For simplicity, the following notations are introduced: $x=\omega, y=i_{q}$ and $z=i_{d}$. Considering system uncertainties and adding the control input,the differential equation (2) is rewritten as

$$
\left\{\begin{array}{l}
\dot{x}=\sigma(y-x)+\Delta f(x, y, z)+u(t) \\
\dot{y}=-y-x z+\gamma x \\
\dot{z}=-z+x y
\end{array}\right.
$$

where $u(t) \in \mathfrak{R}$ is the control input and $\Delta f(x, y, z)$ is an uncertain term, the uncertain part is assumed to be bounded as $|\Delta f(x, y, z)| \leq \delta$

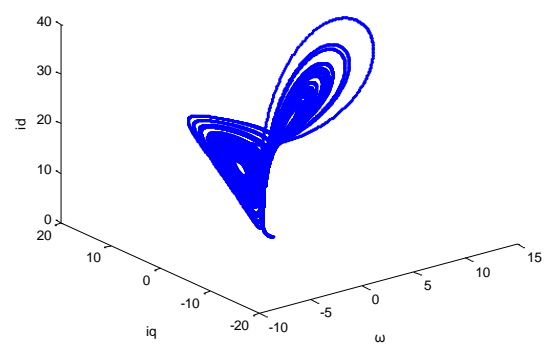

Fig. 1: The chaotic attractors of PMSM

\section{Adaptive Sliding Mode Control Strategy}

\subsection{Adaptive Sliding Mode Controller Design}

The adaptive switching surface is considered as

$$
S(t)=x(t)+\varphi(t)
$$

where $\varphi(t)$ is given by

$$
\dot{\varphi}=\gamma y+\sigma x+\rho x
$$

where $\rho$ is assumed to be an arbitrary positive coefficient.

When the system operates in sliding mode, it satisfies the following equation:

$$
s(t)=x(t)+\varphi(t)=0
$$

Differentiating Eq. (6), leads to the following:

$$
\dot{s}(t)=\dot{x}+\dot{\varphi}=0 \Rightarrow \dot{x}=-\dot{\varphi}=-\gamma y-\sigma x-\rho x
$$

Using Eq. (7), the systems of PMSM can be obtained as

$$
\left\{\begin{array}{l}
\dot{x}=-\gamma y-\sigma x-\rho x \\
\dot{y}=-y-x z+\gamma x \\
\dot{z}=-z+x y
\end{array}\right.
$$

To analyze the stability of equations (8) based on the Lyapunov stability theory. The Lyapunov function candidate is selected as $V=0.5\left(x^{2}+y^{2}+z^{2}\right)$, which leads to

$$
\begin{aligned}
& \dot{V}=x \dot{x}+y \dot{y}+z \dot{z} \\
& =x(-\gamma y-\sigma x-\rho x)+y(-y-x z+\gamma x)+z(-z+x y) \\
& =-(\sigma+\rho) x^{2}-y^{2}-z^{2} \leq 0
\end{aligned}
$$

According to Lyapunov stability theory, it can be concluded that system is asymptotically stable and all state variables will converge to the origin.

To guarantee the system state on sliding mode surface, the equivalent control law is degsigned as following:

$$
u_{e q}=-\gamma y-\sigma x-\rho x-\Delta f(x, y, z)
$$

The reaching law is degsigned as following:

$$
u_{r}=k_{s} \operatorname{sgn}(s)
$$

where $k_{s}$ is the reaching gain, achieved by following adaptive law:

$$
\dot{k}_{s}=-l|s|
$$

where $l$ is a positive constant number.So the control law of PMSM becomes

$$
\begin{aligned}
u & =u_{e q}+u_{r} \\
& =-\gamma y-\sigma x-\rho x-\Delta f(x, y, z)+k_{s} \operatorname{sgn}(s)
\end{aligned}
$$

In order to control chaos in PMSM using Eq. (13), the function $\Delta f(x, y, z)$ must be known. However, in practice 
this function is fully unknown. To overcome this, the control law of PMSM is modified to

$$
u=-\gamma y-\sigma x-\rho x+k_{s} \operatorname{sgn}(s)
$$

\subsection{Stability Analyses}

In order to prove the stability of the scheme presented in (14), the Lyapunov function candidate $\mathrm{V}$ is defined as

$$
V=\frac{1}{2} s^{2}+\frac{1}{2 l}\left(\hat{k}-k_{s}\right)^{2}
$$

The time derivative of (15) is given by

$$
\begin{aligned}
\dot{V} & =s \dot{s}+\frac{1}{l}\left(\hat{k}-k_{s}\right)\left(-\dot{k}_{s}\right) \\
& =s(\dot{x}+\dot{\varphi})+\frac{1}{l}\left(\hat{k}-k_{s}\right)\left(-\dot{k}_{s}\right) \\
& =s\left(\Delta f(x, y, z)+k_{s} \operatorname{sgn}(s)\right)+\frac{1}{l}\left(\hat{k}-k_{s}\right)\left(-\dot{k}_{s}\right) \\
& \leq \delta|s|+k_{s}|s|+\left(\hat{k}-k_{s}\right)\left(-\frac{1}{l} \dot{k}_{s}\right) \\
& \leq \delta|s|+k_{s}|s|+\hat{k}|s|-\hat{k}|s|+\left(\hat{k}-k_{s}\right)\left(-\frac{1}{l} \dot{k}_{s}\right) \\
& \leq(\delta+\hat{k})|s|+\left(\hat{k}-k_{s}\right)\left(-\frac{1}{l} \dot{k}_{s}-|s|\right)
\end{aligned}
$$

Considering that $-\frac{1}{l} \dot{k}_{s}-|s|=0 \quad$, the scalar $\hat{k}$ can be chosen in such a way that the value of $\delta+\hat{k}$ remains negative(i.e., $\delta+\hat{k}=-\eta, \eta>0$ ).Inequality (16) can be rewrited as

$$
\dot{V} \leq-\eta|s|
$$

Therefore, the reaching condition $s(t) \dot{s}(t)<0$ will be satisfied.

\section{Simulation Results}

In order to illustrate the effectiveness of the proposed method, the simulation will be conducted to control chaos in the PMSM drive system under the initial condition of $[x, y, z]^{T}=[3,-4,2]^{T}$ and system parameters of $\sigma=5.45, \gamma=20, \rho=7, l=0.009$. The system is perturbed by an uncertainty term $\Delta f(x, y, z)=0.5-\sin (\pi x) \sin (2 \pi y) \sin (3 \pi z)$. Fig. 2,3 show the state variables of PMSM system when the input control $\mathrm{u}$ is added to the system at the time $t=20$ second.

It can be seen that two control methods all can make the system gradually converge to equilibrium point after the time of 20s. But there is obvious chattering phenomenon using traditional sliding mode method in Fig.2. Convergence speed of proposed method is faster than that of the conventional sliding mode control after the time of 20s. The curve of control signal $u$ is showed in Fig.4.

\section{Conclusion}

In this paper, a adaptive sliding mode control scheme was proposed for eliminating complex chaotic behavior of PMSM. The proposed scheme will give sufficient consideration to uncertainties and external interference in PMSM and guarantee that the state variables of PMSM can converge to equilibrium point from chaotic. Finally, stability analysis will be given based on Lyapunov stability theory. Simulation results are provided to demonstrate the effectiveness and robustness against parameter uncertainties in the chaotic drive system.
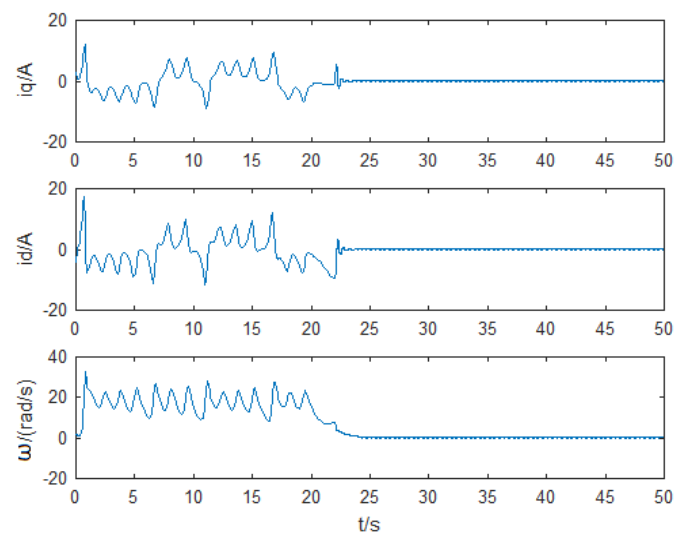

Fig.2: The state variables of PMSM system using traditional sliding mode method
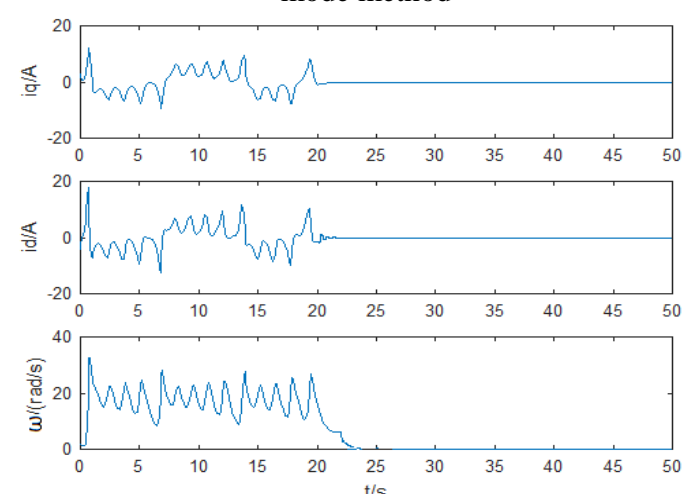

Fig.3: The state variables of PMSM system using proposed method

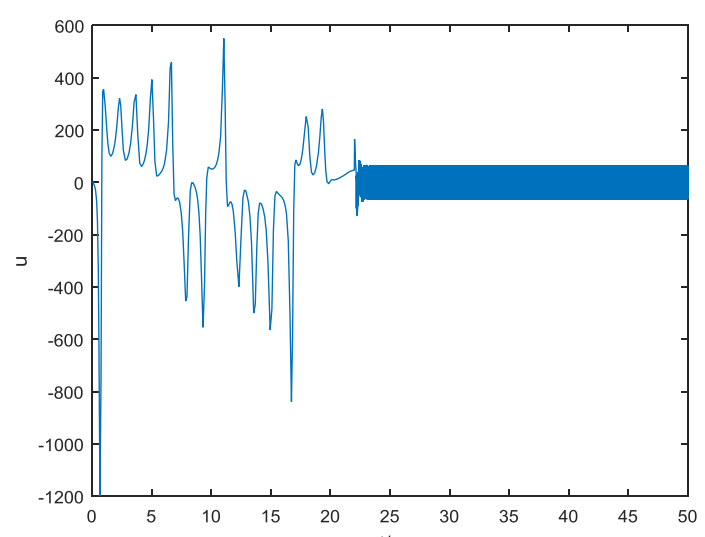

Fig.4:The curve of control signal $u(t)$ 


\section{Acknowledgements}

This work is partially supported by the National Youth Foundation under Grant \#11604224.

\section{References}

[1] Chen Qiang, Nan Yu-Rong, Xing Ke-Xin. "Adaptive sliding-mode control of chaotic permanent magnet synchronous motor system based on extended state observe", Acta Physica Sinica, Vol. 63, No. 22, pp. 1$8,(2014)$

[2] Tong Yaonan, Li Chunlai, Gheng Wangbin. "Controlling Chaos in Permanent Magnet Synchronous Motor Based on Tridiagonal Matrix Stability Theory", International Workshop on Intelligent Systems\&Application, pp.1-4, (2011).

[3] Ma Caoyuan, Wang Longshun. "Sliding mode control of chaos in the noise-perturbed permanent magnet synchronous motor with non-smooth air-gap", Mining Science and Technology, 21( 6),pp. 835-838, (2011).

[4] Zhang Xuemei,Li Qiuming. "Higher-order nonsingular terminal-sliding-mode observer for permanent-magnet synchronous motor", Control Theory\&Applications, 28,(10),pp.1467-1472, (2011).

[5] Haipeng Ren, Ding Liu. "Nonlinear Feedback Control of Chaos in Permanent Magnet Synchronous Motor", IEEE TRANSACTIONS ON CIRCUITS AND SYSTEMS, 53(1),pp. 45-50, (2006).

[6] Xi Xiao, Changming Chen. "Dynamic Permanent Magnet Flux Estimation of Permanent Magnet Synchronous Machines", IEEE TRANSACTIONS ON APPLIED SUPERCONDUCTIVITY, 20(3),pp. 10851088, (2010).

[7] Wei Xiang, Yugao Huangpu, "Second-order terminal sliding mode controller for a class of chaotic systems with unmatched uncertainties", Commun Nonlinear Sci Numer Simulat, 15(11),pp. 3241-3247,(2010).

[8] Juntao Li, Wenlin Li. "Sliding mode control for uncertain chaotic systems with input nonlinearity", Commun Nonlinear Sci Numer Simulat, 17(1),pp. 341348, (2010).

[9] Wei Xiang, Fangqi Chen. "An adaptive sliding mode control scheme for a class of chaotic systems with mismatched perturbations and input nonlinearities", Commun Nonlinear Sci Numer Simulat, 16(1),pp.1-9, (2011).

[10] Shi Fatao, Ma Yumei. “Adaptive Fuzzy Control for Chaotic Permanent Magnet Synchronous Motor Based on State Observer". JOURNAL OF QINGDAO UNIVERSITY, 29(4),pp. 33-39, (2014).

[11] Mohammad Pourmahmood Aghababa, Sohrab Khanmohammadi. "Finite-time synchronization of two different chaotic systems with unknown parameters via sliding mode technique", Applied Mathematical Modelling, 35(6),pp.3080-3091, (2011).

[12] Seong-S. Kim, Han Ho Choi. "Adaptive synchronization method for chaotic permanent magnetsynchronous motor", Mathematics \& Computers in Simulation, 101(7),pp. 31-42, (2014).

[13] CHEN Xiangqing, WANG Jiankui. "Finite-time control of chaos in permanent magnet synchronous motor", Proceedings of the 32nd Chinese Control Conference, pp.26-28, (2013).

[14] D. Wei, and B. Zhang, "Controlling chaos in permanent magnet synchronous motor based on finite-time stability theory", Chinese Physics B, 18(4),pp. 1399 - 1403, (2009).

[15] Yezhan Zeng. "Adaptive Sliding Mode Control for Permanent Magnet Synchronous Motor".2011 International Conference of Information Technology, pp. 356-358,(2011). 